\title{
An Open and Extensible Service Discovery for Ubiquitous Communication Systems
}

\author{
Nor Shahniza Kamal Bashah ${ }^{1}$, Ivar Jørstad ${ }^{2}$, and Do van Thanh ${ }^{3}$ \\ ${ }^{1}$ Norwegian University of Science and Technology, Department of Telematics, \\ O.S. Bragstads Plass 2E, NO-7491 Trondheim, Norway \\ nor@item.ntnu.no \\ ${ }^{2}$ Ubisafe, Gamleveien 252, 2624 Lillehammer, Norway \\ ivar@ubisafe.no \\ ${ }^{3}$ Telenor \& NTNU, Snaroyveien 30, 1331 Fornebu, Norway \\ thanh-van.do@telenor.com
}

\begin{abstract}
In future ubiquitous communication systems, a service can be anything and introduced by anybody. Consequently, same or equivalent services may have different names and services with same name or type may be completely different. Existing service discovery systems are incapable of handling these situations. We propose a future service discovery, which is able to discover all these new service types. In addition, it is capable to find services that are not exact matches of the requested ones. More semantics are introduced through attributes like EquivalenceClass, ParentType and Keywords.
\end{abstract}

Keywords: Service advertisement, service discovery, service lookup, service matching, service registration.

\section{Introduction}

In this research, a future service discovery system is proposed to solve the problem of discovering services in a ubiquitous communication environment, where similar services can have different names in different languages and services with same name may not offer the same functions and capabilities. The requirements on future service discovery systems are derived and more details can be found in [1]. The future service discovery system consists of service registration, service advertisement and request and service matching.

For the service registration the service provider must supply the details about the service that are necessary to detect, identify and use the service as follows:

- Service name - to denote and recognise the service. We propose to extend the service name format of future service discovery to allow any language.

- Service type - to carry on a search and identification of the functions offered by the service. There are three options of service type which are:

o Brand-new service type - without relation with any existing service type. Hence the ParentType has to be set to nil and the EquivalentClass is then left empty. 
o Equivalent service type - the service type has a different name and may be another implementation but is equivalent to an existing service type. The service provider has to add the name of the existing service type and also all the known service type in different languages.

o Subtype of an existing service type - The service type has all the functions and features of an existing service type but has also additional ones. The ParentType is hence indicated.

There are two alternatives for implementing service advertisement:

- The service registry or broker can take the initiative and broadcast the list of available services periodically.

- A client wanting to find services issues a request for a list of available services to the service registry.

However, both alternatives have their own advantages and disadvantages hence, selection of appropriate alternatives has to be considered and empirical methods have to be used. Anterior service advertisement may be a solution to be considered. Service advertisement can be made before the mobile user enters the location thus contribute to speeding up the service discovery and ensuring service continuity.

Instead of asking for the list of available services and carrying the service matching itself the client can request for a particular service or set of services. However, they may not have the opportunity to discover new and unknown services.

The service matching can be performed by the mobile client after the acquisition of the list of available services or by the network system upon receipt of a service request. We propose to extend the match operation on the Equivalence class, Service subtyping and Keywords of the requested service type.

\section{Conclusions}

This research explains the necessity of an innovative service discovery capable of handling similar services with different name and different services with same name. A prototype is currently under development and will be accomplished in the near future. Next, the experiments with various number of clients, number of services, different bandwidths and complicated service ontology will be carry out. The collected results will then be used to optimise the service discovery prototype. Another relevant and exciting future work will be the contribution to the extension of the 802.21 MIHF (Media Independent Handover Function) [2] for devices equipped with multiple wireless access technologies. Such an extension will ensure service continuity across multiple heterogeneous access networks.

\section{References}

1. Kamal Bashah, N.S., Jørstad, I., van Do, T.: Service Discovery in Future Open Mobile Environments. In: Proc. the Fourth International Conference on Digital Society (ICDS 2010), February 10-16, St. Maarten, Netherlands Antilles (2010), ISBN: 9780769539539

2. Media Independent Handover Services, Draft IEEE Standard for Local and Metropolitan Area Networks: Media Independent Handover Services, IEEE P802.21/D7.1 (August 2007) 